\title{
THE ROLE OF BUBBLE-SIZE EQUILIBRATION IN THE TRANSIENT BEHAVIOR OF FISSION GAS
}

\author{
by
}

E. E. Gruber

BASE TECHNOLOGY

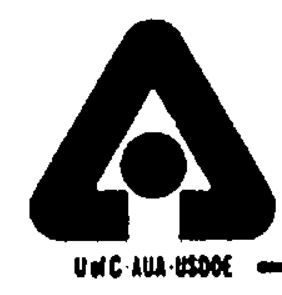

ARGONNE NATIONAL LABORATORY, ARGONNE, ILLINOIS

Prepared for the U. S. DEPARTMENT OF ENERGY

under Contract W-31-109-Eng-38 

TABLE OF CONTENTS

$\underline{\text { Page }}$

ABSTRACT. ....................................... 7

I. INTRODUCTION.................................. 8

II. ANALYSIS. .................................... 12

A. Rate of Bubble-size Equilibration................. 12

B. Relative Importance of Equilibration.................. 13

c. Incorporation of Equilibration in the FRAS Code.......... 18

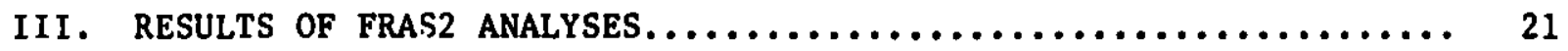

A. Equilibration Effects in the H3 Transient.............. 21

B. Ef fects of Equilibration on Gas Behavior............... 22

C. Dispersive Potential of Nonequilibrium Bubbles............ 26

IV. SURMARY AND CONCLUSIONS........................... 29

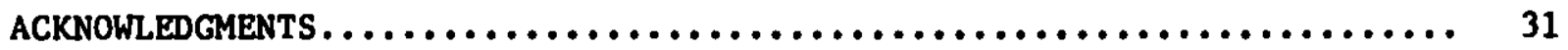

REFERENCES. ........................................ 31 


\section{LIST OF FIGURES}

No.

Title

Page

1 Expansion of a 10-nm Bubble for Various Thermal Ramps......... 14

2 Expansion of a 100-nm Bubble for Various Thermal Ramps........ 14

3 Shrinkage of a 10-nm Bubble for Various Cooling Rates......... 15

4 Shrinkage of a 100-nm Bubble for Various Cooling Rates........ 15

5 Bubble Equilibration Following Coalescence of Two

$10-n$ Bubbles at $2273 \mathrm{~K} . \ldots \ldots \ldots \ldots \ldots \ldots \ldots \ldots \ldots \ldots \ldots \ldots \ldots \ldots$

6 Relaxation Times for Coalescence-product Bubbles as

Functions of Temperature.............................. 18

7 FRAS Results for the TREAT H3 Test, Assuming Different

Surface-diffusion Coefficients and Heats of Transport......... 21

8 FRAS2 Results for the TREAT H3 Test, Assuming Different

Volume-diffusion Coefficlents......................... 22

9 Comparison of Cumulative Bubble-size Distributions at the

Solidus for Three Linear Thermal Ramps, Using FRAS

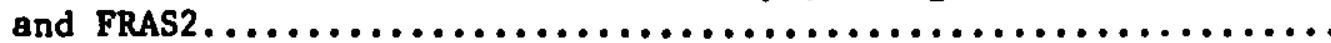

10 Cumulative Bubble-size Distributions Calculated with the

FRAS and FRAS2 codes for a 500-K/a Heating Rate..............

11 Gas Release to Grain Bound.: :es for Three Heating Rates,

Calculated with the FRAS Code..........................

12 Gas Release to Grain Boundaries for Three Heating Rates,

Calculated with the FRAS2 Code........................

13 PRAS-calculated Intragranular Swelling for Three

heat Ing Rates.....................................

14 FRAS2-calculated Intragranular Swelling for Three

Heat Ing Rates..................................... 
LIST OF TABLES

No.

Title

Page

1

Results of Heating-rate Comparisons................

24

2

Dispersive-potential Results from FRAS2 Calculations....... 

THE ROLE OF BUBBLE-SIZE EQUILIBRATION IN THE

TRANSIENT BEHAVIOR OF FISSION GAS

by

E. E. Gruber

\section{ABSTRACT}

An explicit analysis of bubble-size equilibration by volume diffusion has been derived and incorporated into the FRAS2 code. This code was developed for mechanistic analysis of transient fission-gas behavior in LMFBR fuel, but had been limited by the approximation that the time required for bubble-size adjustment, following coalescence or changes in temperature or pressure, was negligible.

The equilibration phenomenon is fllustrated by several Idealized examples. If the fuel temperature rises Iinearly with time, an isolated bubble will expand rapidiy toward its equilibrlum size only after the temperature reaches a critical value that depends on both bubble size and heating rate. Conversely, If the temperature is reduced unfformly, an 1solated bubble will shrink only until diffusion becomes too slow for further size change. The final size that is "frozen in" may be significantly larger than the final equilibrlum size. Finally, 180thermal equilibration following coalescence of two equal bubbles can be described conventently in terms of relaxation times, which depend on bubble size and temperature.

However, a complete picture of the role of equilibration in transient f1ssion-gas behavior can only be gained through an Investigation of the bubble-size distribution and Its evolution. Such an Investigation 18 carried out with the FRAS2 code, in which the explicit equilibration model is 1mplemented. Since nonequilibrlum bubbles in a thermal transient are generally overpressured, the gas represents a source of energy that can concelvably dierupt the fuel. This "dieperalve potential" 18 also calculated in the FRAS2 code. 


\section{INTRODUCTION}

Migration and coalescence of fission-gas bubbles are important phenomena In the transient behavior of nuclear fuels. An understanding of these fundamental processes is essential to develop useful treatments of the effects of fission gas on fuel swelling and disruption during thermal transients.

Since both migration and coalescence rates depend on bubble size, it is necessary to characterize this size accurately in modeling calculations. In most analyses, the bubble radius $r$ is assumed to satisfy the equilibrium relation $r=2 \gamma /\left(p-p_{h}\right)$, where $\gamma 1 s$ the surface tension and $p-p_{h}$ is the excess pressure in the bubble compared to the hydrostatic stress in the surrounding medium.

When tiwo bubbles coalesce, however, two distinct processes occur, essentially consecutively. First, there is a conversion from a double-bubble configuration to a larger single bubble with the same total volume. This step occurs, in the case of small bubbles ( $₹ ₹ 1 \mu \mathrm{m}$ ), primarily by the surfacediffusion migration of atoms driven by capillarity effects. Other mechanisms could also contribute to this morphology change, depending on conditions: volume diffusion, evaporation-condensation, and even viscous flow or creep. The initial result of this first step is a spherical bubble of the same volume as the initial bubble pair. Nichols has shown that the driving force for this step is the decrease in surface energy because of the net reduction in surface area, and that coalescence should occur for all practical cases.

The second process is one of volume adfustment. Since for instantaneous coalescence the gas pressure 18 unchanged, while $r$ is increased, the surfacetension restraint $2 \gamma / r$ lo not suffictent to balance the gas pressure, and the bubble will tend to expand. Th1s expansion tast take place by long-range migration of atoms or volume diffusion (although creep may contribute in some cases). The process of volume adjustment 18 always thermodynamically favorable and is driven by the free energy change of the gas within the bubble. N1chols has pointed out, however, that volume adjustment 18 much slower than coalescence, because of both a lower driving force and a slower transport mechanism. Th1s difference in rates, in fact, 18 the feature that makes it convenient to consider coalescence and size adjustment as occurring consecutively, rather than simultaneously. 
These considerations were at least qualitatively recognized even before N1chols' analysis. However, because of the difficulty in incorporating the volume-equilibration process into modeling of fission-gas behavior, it has generally been assumed that coalescence and equilibration both occur Instantaneously. ${ }^{2}, 3$ This assumption has come into question recently as modeling efforts have matured and as the experimental data base has broadened.

The general effect of nonequilibrlum on transient fission-gas behavior is qualitatively simple: the bubbles do not expand as rapidly as predicted by Instantaneous equilibration models when temperature increases, pressure decreases, or coalescence occurs. As a result, the bubbles migrate faster, causing enhanced release, and contribute less to swelling. A quantitative assessment is not so simple, however. Coalescence probabilities also depend on bubble sizes, both through the effect on migration velocity or diffusivity, and through the effect on the cross sections for collisional coalescence.

In an attempt to include equiifbration effects in the modeling of translent fission-gas behavior, Esteves ${ }^{4}$ developed a two-varlable multigroup numerical approximation to describe the evolution of the bubble population. This work was extended by others, ${ }^{5}$ and applied to determine the Importance of nonequilibrium bubbles. A small but significant enhancement of fission-gas release for the TREAT H3 transient ${ }^{6}$ was predicted by the calculations. Other features that were predicted included a "freezing in" of the bubble-size distribution during cooling and a reduction in swelling. The detalled twovarlable treatment, however, leads to increased complexity and computer atorage requirements.

The steady-state GRASS code also treats bubbles as growing to equilibrium size Instantaneously following coalescence. When GRASS results were compared to experimental data, it was found that a modification was needed to describe the limited rate of growth of coalescing bubbles. In an effort to keep computational requirements small, a simple modification was developed by J. Rest. 7 The rate at which bubbles grow from the 1 to the $1+1$ class is now reduced from the calculated rate by a relaxation-time factor, which depends on the bubble size as well as the energles of formation and migration of vacancies.

C. Ronch1 eugsested a more comprehensive procedure by which the rate of growth of nonequilibrium bubbles could be included explicitly in transient calculatione with the GRASS code. ${ }^{8}$ That analyo18, which apparently has not 
been Implemented, is based on the assumption that the force on a vacancy depends on the varlation in the radial stress component with distance from the bubble.

In another analysis of the equilibration phenomenon, W. Wang and $R$. Singh have analyzed equilibration by primary creep. 9 Vacancy diffusion was also considered in their work, but the driving force for vacancy diffusion was considered to be the equivalent stress at the bubble surface.

If equilibration were assumed to result from vacancy diffusion caused by stress effects, the most plausible driving force for vacancy diffusion would be the variation in hydrostatic stress with distance from the bubble. However, for an elastic continuum, the hydrostatic stress does not vary with distance from a bubble. The modification to the FRAS code ${ }^{3}$ is not based on stress considerations. Instead, vacancy diffusion is considered to result from concentration gradients that develop around bubbles. The thermodynamic approach is used to establish boundary conditions, following Greenwood et al. ${ }^{10}$ Minimization of the free energy change with respect to changes in bubble radius provides the equilibrium condition. The apparent driving force for equilibration that results can be expressed in terms of pressures, but does not derive from a mechanical analysis.

The motivation for the development of this treatment and 1 ts incorporation into the FRAS2 code ${ }^{11}$ arose from the results of comparisons of experimental data with FRAS-code predictions. The experimental data included results from direct-electrical-heating (DEH) tests ${ }^{12}$ and from FGR tests. ${ }^{13}$ Although good agreement was obtained with the FRAS code in some cases, the bubble size was greatly overpredicted in others. In particular, 1t appeared that the discrepancy was greatest for rapld translents for which large bubbles were predicted, and was a direct consequence of ignoring the equilibration time in the calculations. 11

The equ1libration treatment applied in FRAS2 was developed as a compromise; equilibration 18 calculated explicitly, as in the work by Esteves, ${ }^{4}$ but only an average "nonequilibrium" is considered for each size class. The expl1c1t modeling is accomplished by assuming that coalescence inftially conserves volume and that equilibration in each size class proceeds at a rate determined by volume diffusion. The modeling approach will be described in the next section. The difference between the actual and equlibrium bubble $81 z e s$ can be calculated to show in detall the effects of nonequlibrium on the evolution of 
the bubble-size distribution, on release of gas from the grains to grain boundaries, and on intragranular swelling. These results will be described 1n Sect. III. 


\section{Rate of Bubble-size Equilibration}

The basic analysis of the rate at which the bubble volume changes was Iven in an early paper in the field of bubble physics. 10 The expression deIved for the vacancy concentration at equilibrlum near a bubble surface, based I free energy considerations, is

$$
c^{v}=c_{e}^{v} \exp \{-[p-(2 \gamma / r)] \Omega / k T\}
$$

here $c_{\mathrm{e}}^{\mathrm{v}}$ is the equilibrium vacancy concentration, $\Omega$ is the molecular volume of he solid, $k$ is Boltzmann's constant, and $T$ 1s the absolute temperature. The emperature of the gas 18 assumed to be the same as the fuel temperature. The iffusion equation in spherical coordinates:

$$
\frac{d^{2} c^{v}}{d \rho^{2}}+\frac{2}{\rho} \frac{d c^{v}}{d \rho}=0,
$$

as solved to determine the vacancy flux at the bubble surface, $\rho=r$, and lence the rate of change of bubble radius. Arguments concerning fission acancies and interstitials were introduced, but thelr contributions were conidered small in deriving the end result:

$$
\frac{d r}{d t}=D_{v} c_{e}^{v}\left(p-\frac{2 \gamma}{r}\right) \frac{\Omega}{r k T},
$$

here $D_{v}$ is the vacancy diffusion coefficient. Several assumptions were made .n the derivation of Eq. (3). First, the exponential in Eq. (1) was assumed to ie small. Second, the bubble separation $2 R$ was assumed to be much greater than :he bubble radius $r$. Finally, the vacancy concentration at $R$ was taken to be :he equilibrium concentration.

A more general expression that includes the effect of local hydrostatic sressure and the effect of bubble separation, and does not use an approximation :or the exponent1al, has been derived for the present application:

$$
\frac{d r}{d t}-D_{u} \frac{R}{r(R-r)}\left\{1-\exp \left[-\left(p-P_{h}-\frac{2 \gamma}{r}\right) \frac{\Omega}{k T}\right]\right\},
$$


where $D_{u}$ has been substituted for $D_{v} c_{e}^{v}$, since uranium self-diffusion is ratecontrolling. The correlation coefficient is taken to be unity for the present.

There is one difficulty with Eq. (4) that must be considered. As the bubble separation decreases, the equilibrium rate appears to increase. The reason for this is the assumed boundary condition that the equilibrium vacancy concentration is maintained at $R$ even when $R$ - $r$ becomes very small. A more realistic equation results from the simplification used by Greenwood et al. 10 based on the assumption that $R \gg r$. The physical implication is that the gradient in vacancy concentration at the bubble surface is the same as it would be if the bubbles were widely separated. The vacancy concentration between close, nonequilibrium bubbles would consequently be reduced from the equilibrium value. The general result is then

$$
\frac{d r}{d t}=\frac{D_{u}}{r}\left\{1-\exp \left[-\left(p-p_{h}-\frac{2 \gamma}{r}\right) \frac{\Omega}{r T}\right]\right\} \text {. }
$$

The degree of nonequilibrium is indicated by the term in parentheses in this equation. When a bubble is formed by the coalescence of two bubbles of equal radius $r$, the radius increases very quickly to $2^{1 / 3} r$; $\mathrm{dr} / \mathrm{dt}$ is then positive. Similarly, if the temperature rises, $p$ increases, and agaln $\mathrm{dr} / \mathrm{dt}$ is positive. A decrease in hydrostatic pressure has the same effect.

\section{B. Relative Importance of Equilibration}

Although it is difficult to characterize the conditions under which equl1bration is important, some simple illustrations can be given as an aid to understanding the phenomenon. The more general application of the analysis, In the PRAS2 code, w111 be considered in the next subsection.

Consider first a constant-temperature ramp: $\mathrm{dT} / \mathrm{dt}=\mathrm{A}$. An equilibrium bubble at some Inftial temperature, say $100 \mathrm{~K}$, w11l tend to expand as the temperature rises. However, the rate of expansion will be negligibly small unt1l the temperature $18 \mathrm{high}$ enough for rapid diffusion. A almple computer program was developed to apply Eq. (5), with material properties for mixed oxide and $P_{h}=0$, to this situation, using finite-difference methods. The bubble size 18 plotted In P18. 1 for an inftial bubble radius of $10 \mathrm{~nm}$, and in F18. 2 for an Initial radius of $100 \mathrm{~nm}$, as functions of temperature for temparature up to the oolidue of mixed-oxide fuel. In both figures, the equilibrium 

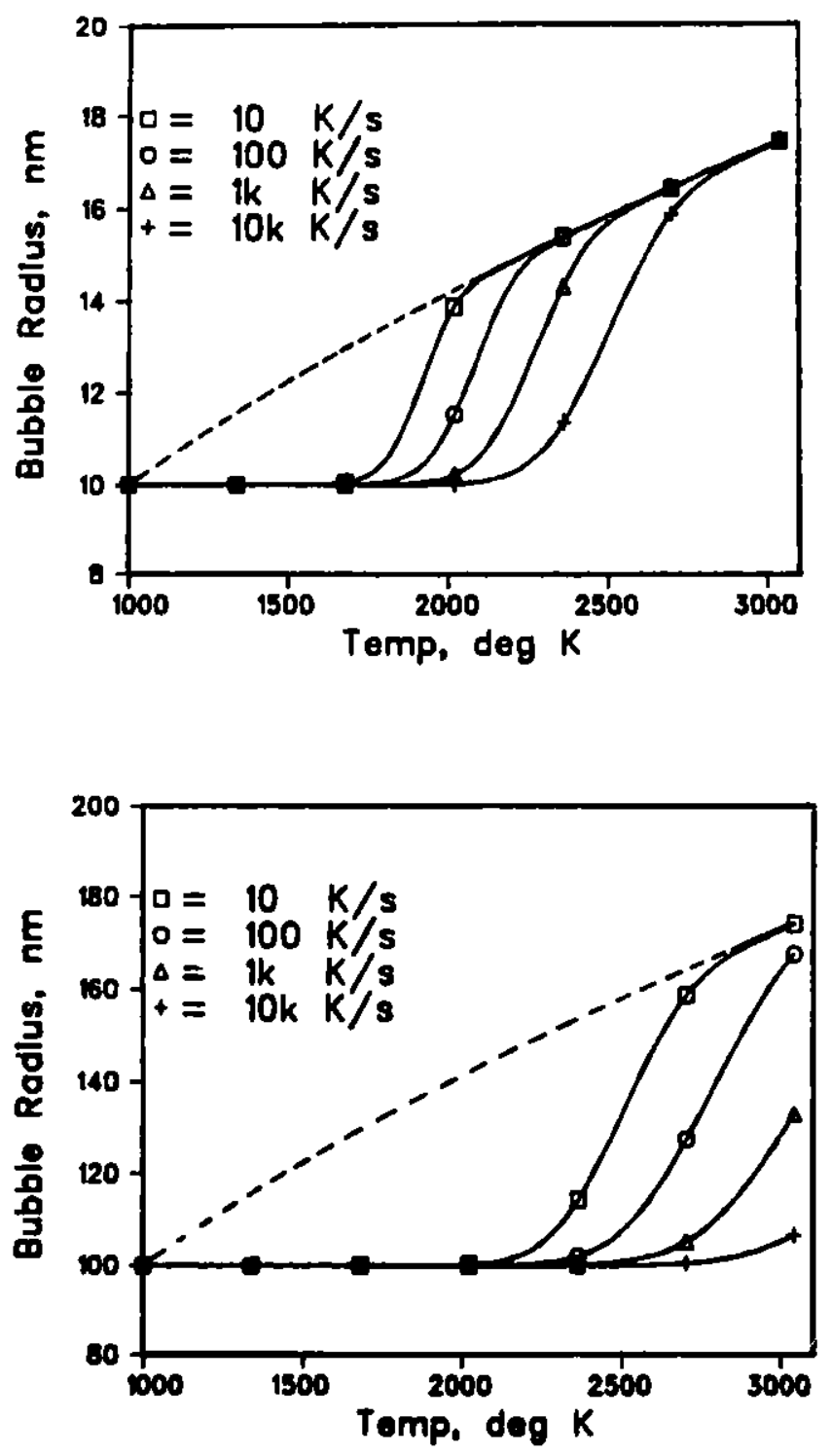

Fig. 1.

Expansion of a 10-nm Bubble for Various Thermal Romps.
Fig. 2. Expansion of a 100-rm Bubble for Various Thermal Romps.

bubble radius 18 shown by a dashed line, and the calculated radius dependence 1s shown for several thermal ramp rates. The results show a very rapid expansion occurring near a critical temperature that depends on heating rate and on bubble $812 e$.

A related phenomenon 18 the "freezing In" of bubble sizes during cooling, discussed by Grelsemeyer et al. ${ }^{5}$ Th1s same analysis has been applied for negative temperature ramps to study bubble shrinkage during cooling. The results are shown in Fig. 3 for a 10-n Init1al bubble radius, and in F18. 4 for a 100nm bubble. At high temperatures, equilibrium 18 malntalned, but as the temperature decresses, as asymptotic size 18 attained that depends, again, on cooling rate and Inttial bubble size. This size can also be characterized 

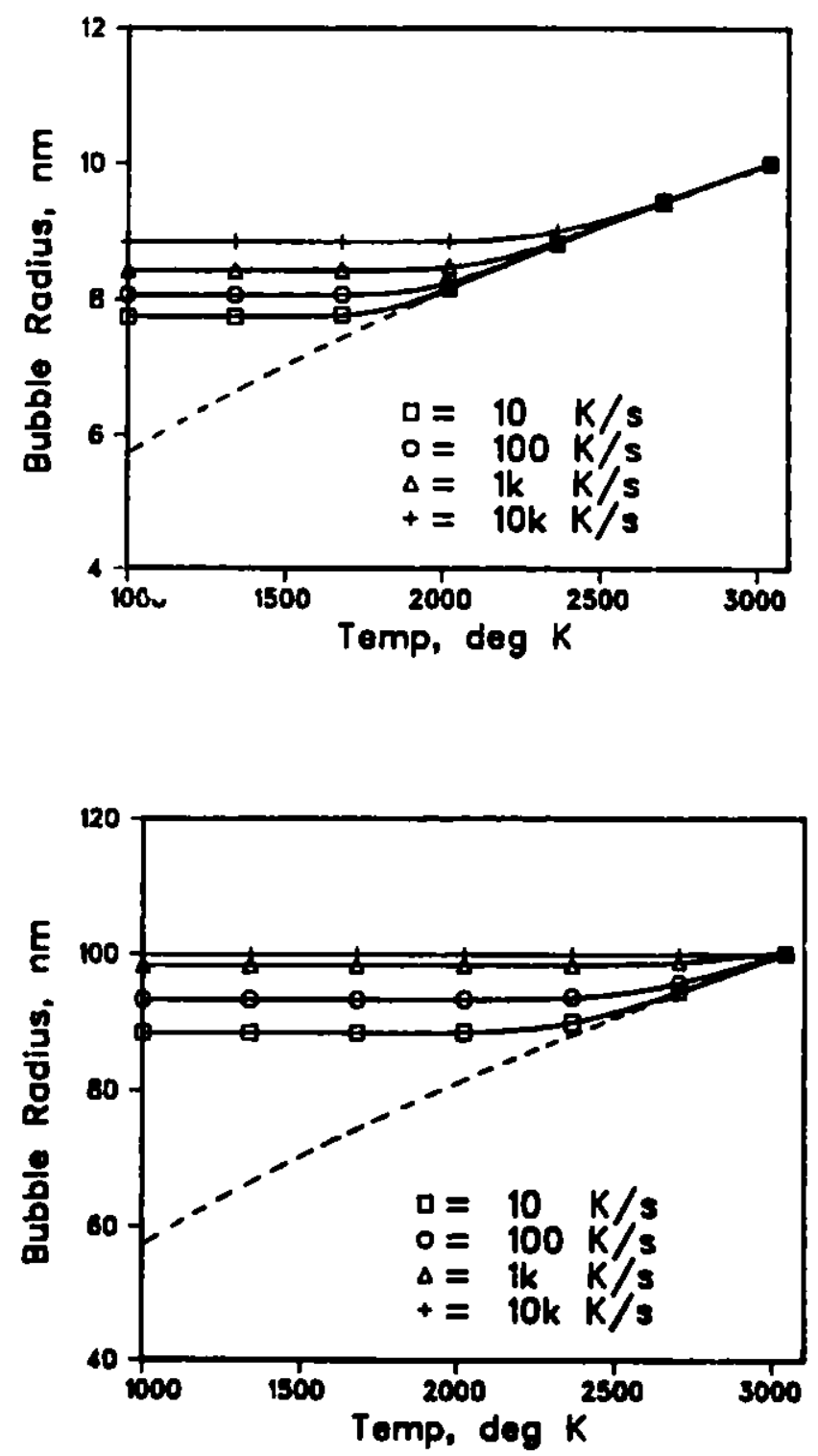

Eig. 3.

Shrinkage of a 10-nm Bubble for Various Cooling Rates.
Fig. 4.

Shrinkage of a 100-ron Bubble for Various Cooling Rates.

by a critical temperature, as was done by Greisemeyer et al. for a more general distribution of bubble sizes. The fact that the "critical temperature" Is only an approximation when a range of bubble sizes 18 considered was not noted by Grelsemeyer et al., but 18 apparent from a comparison of F1g8. 3 and 4 .

The preceding discussion gives some indication of the dominance of diffusion kinetics in bubble equilibration for an 18olated bubble. Another 1uportant eituation 18 the equilibration of the product bubble formed by the coalescence of two smaller bubbles. This situation can also be treated in a simplified way to develop an understanding of the conditions for which equilibration 1s Important. 
The relaxation-time concept provides a convenient, quantitative description of the effects of the parameters involved in equilibration. An exponential decay of some parameter with time is implied in the definition of a relaxation t1me $\tau$. The process considered here 18 the varlation of the radius of a product bubble formed at $t=0$ by the coalescence of two 1dentical bubbles of radius $r_{1}$. The product-bubble radius $r$ varies from an initial value $r_{0}$ toward an asymptotic value $r_{\text {eq }}$. If the equilibration were a true exponential decay, the variation in $r$ would be described by

$$
r=r_{0}+\left(r_{e q}-r_{0}\right)\left(1-e^{-t / \tau}\right)
$$

Differentiation of $\mathrm{Eq} .(6)$ gives

$$
\frac{d r}{d t}=\frac{r_{e q}-r_{0}}{\tau} e^{-t / \tau}
$$

The relaxation time $\tau$ can consequently be defined from the initial slope, by setting $t=0 \mathrm{ln} \mathrm{Eq.} \mathrm{(7)} \mathrm{and} \mathrm{solving} \mathrm{for} \tau$ :

$$
\tau=\frac{r_{e q}-r_{0}}{(d r / d t)_{t}=0} .
$$

The actual variation of $r$ is described by Eq. (4), which can be simplified for the present purpose by assuming that $P_{h}=0$ and that the gas is 1deal. The simplified form of Eq. (4) then becomes

$$
\frac{d r}{d t}=\frac{D_{u}}{r}\left\{1-\exp \left[-\frac{4 \gamma \Omega}{r k T}\left(\left(r_{1} / r\right)^{2}-0.5\right)\right]\right\}
$$

Although Eq. (9) 18 hardly of the same form as Eq. (7), the varlation of $r$ with $t$ w1ll be shown to be very simflar to that described by Eq. (7).

Equation (9) can be simplified further by noting that, for $\gamma=0.626 \mathrm{~J} / \mathrm{m}^{2}$, $\Omega=4.08 \times 10^{-20} \mathrm{~mm}^{3}$, and $T=2300 \mathrm{~K}$, we have $4 \mathrm{r} \Omega / \mathrm{kT}=3.23 \mathrm{~nm}$. Since the variation of $\left(r_{1} / r\right)^{2}$ ranges from 0.5 to 0.63 , it follows that, for $r \geqslant 4 \mathrm{~nm}$, the exponential factor $18 \% 0.1$. Expansion of the exponential and neglect of h1gher-order terms ylelds the approximate result

$$
\frac{d r}{d t}=\frac{4 D_{u} m}{r^{4} k T}\left(r_{1}^{2}-0.5 r^{2}\right)
$$


At $t=0$, we find $r=1.2599 r_{1}$; the initial slope is therefore

$\left(\frac{d r}{d t}\right)_{t=0} \cong \frac{0.3275 \gamma \Omega D_{u}}{r_{1}^{2} k T}$.

From this result and the definition of $\tau$ in Eq. (8) we obtain the general result

$\tau \cong 0.471 \mathrm{r}_{1}^{3} \mathrm{kT} / \gamma \Omega \mathrm{D}_{\mathrm{u}}$

Equation (12) can be applied to quantitative examples by substituting the matertal parameters mentioned above and the self-diffusion coefficient ${ }^{14}$

$D_{u}=1.9927 \exp (-55600 / T) \mathrm{mm}^{2} / \mathrm{s}$

to obtain

$\tau \cong 1.275 \times 10^{-18} \mathrm{r}_{1}^{3} \mathrm{~T} \exp (55600 / \mathrm{T}) \mathrm{8}$

A simple finite-difference approach was used to solve Eq. (9) for the bubble radius as a function of time, using $T=2273 \mathrm{~K}$ and $r=10 \mathrm{~nm}$. The results are represented in Fig. 5 by the symbols.

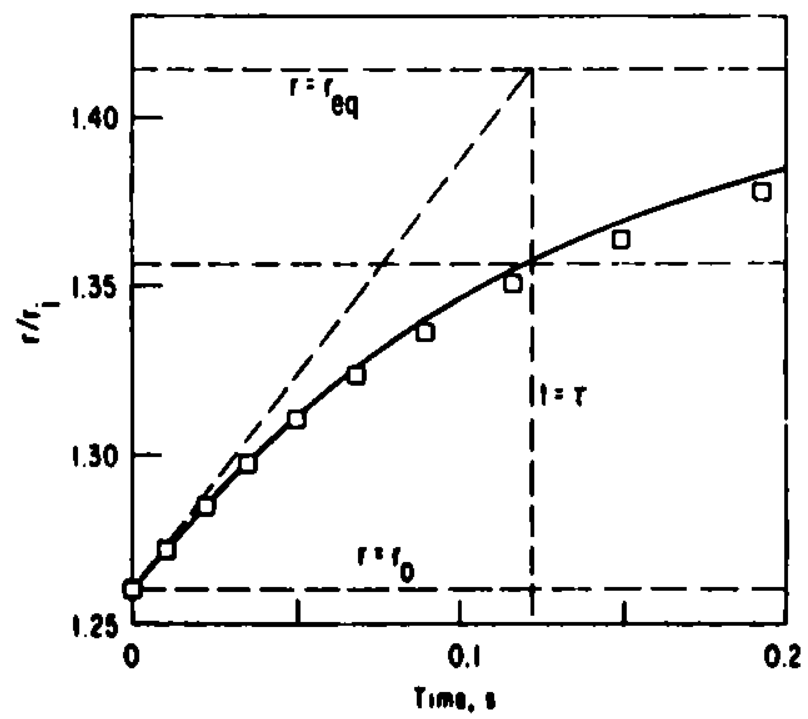

Fig. 5. Bubble Equilibration Following Coalescenos of Two 10-m Bubbles at $2273 \mathrm{~K}$. 
A normalized form of $\mathrm{Eq} .(6)$ :

$$
r / r_{1}=1.2599+0.1543\left(1-e^{-t / \tau}\right),
$$

was also solved for the same example, using $\tau=0.1216 \mathrm{~s}$, calculated from Eq. (14). The results are shown by the solid lire in Fig. 5. At $t=\tau$, the difference between $r$ and $r_{e q}$ has decayed to $1 / 6$ times the intitial difference. The discrepancy in the results by the two methods is small; the use of the Inftial slope from Eq. (11) to define the relaxation time is therefore a good approximation.

Equation (14) has been applied to a range of conditions to demonstrate the influences of bubble radius and temperature on relaxation time. The results, shown in Fig. 6, Indicate that smaller bubbles should equilibrate quickly

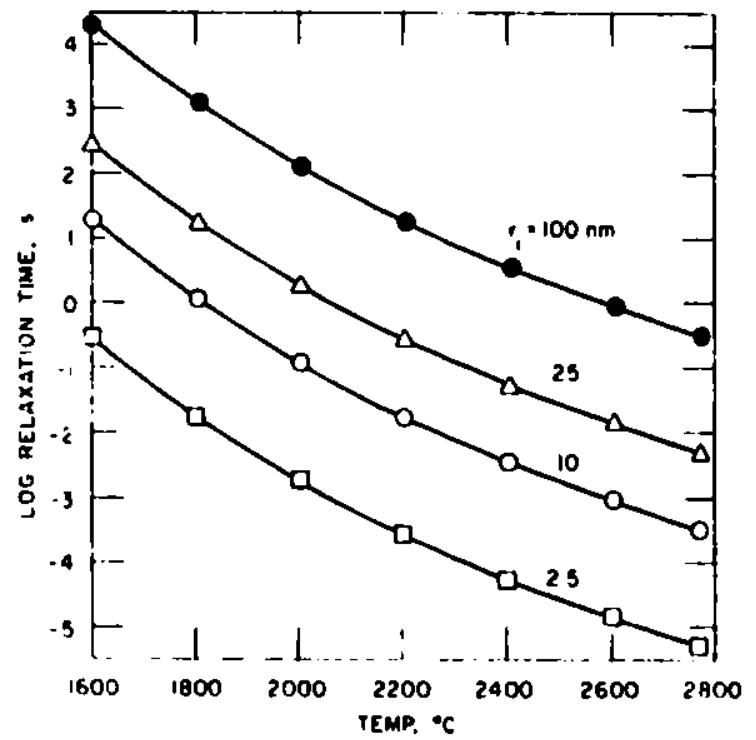

Fig. 6. Relaxation Times for Coalescence-product Bubbles as Functions of Temperature. at sufficiently high temperatures. However, problems arise in transient modeling when gas concentrations are sufficiently high that the mean bubble size (and therefore the fuel swelling) becomes large. Larger bubbles may require a relaxation time significant in comparison to the transient time scale. Since bubbles may not reach equilibrium before again coalescing, subsequent product bubbles may deviate further from equilibrlum. These more complex considerations are better analyzed in the more general context of the FRAS code.

\section{Incorporation of Equilibration in the FRAS Code}

Consideration of the above discussion of equilibration effects leads to the conclusion that a general treatment ls complicated. Temperature, pressure, coalescence, and bubble sizes must all be considered. Since the analysis carried out by the FRAS code includes all of these factors, 15 it is a logical Btep to incorporate equilibration explicitly into the code. 
This step is conceptually simple, although its accomplishment in the code required extensive revision. The method can be summarized by the following steps: (1) Migration and coalescence are calculated as before, except that the nonequilibrium bubble size is used to calculate migration velocity and coalescence probability. (2) Volume is conserved upon coalescence. The volume jer bubble is averaged over all bubbles in each size class. (3) All bubbles in each class are assumed to equilibrate at the same rate, determined by the average conditions in the class.

Separation of the coalescence and equilibration steps requires some justification, since both occur simultaneously in each time step. The time step is selected internally in a manner that precludes a large change in the population of a given size class in one time step. The average degree of nonequilibrium of any size class is therefore not significantly altered by coalescence in a time step, and most of the bubbles in a given class are affected only by equilibration during the step. It is therefore appropriate to evaluate the equilibration continuously over each time step. The validity of this approach is supported by preliminary analyses that Indicate no significant effect of time-step size within the normal range of sizes.

A more important limitation enters in the assumption that the degree of nonequilibrium in each size class can be adequately represented by the average volume per bubble. Each size class is characterized by the mean number of gas atoms per bubble. Each coalescence results in the loss of two bubbles, with their associated numbers of gas atoms and bubble volumes, from their size classes, and the addition of one bubble, containing the same total number of gas atoms and bubble volume, to a product-bubble class. In practice, the product bubble 18 aplit fracticnally between two adfacent classes so that both gas atoms and bubble numbers can be properly conserved. Th1s procedure 1s necessary because only a fintte number of size classes is chosen. The product bubble rarely contains the exact number of atoms used to represent a size class and w111 generally contribute less than the average volume per bubble in its new s1ze class. Nevertheless, the volumes of added bubbles are averaged with the volumes of existing bubbles in the size class, to provide an average volume per bubble, from which a new, nonequilibrlum, average radiug is calculated for the 8128 class. 
The equilibration is calculated for each time step and size class accordIng to $\mathrm{Eq}$. (5), using a simple finite-difference treatment. The coalescence time step is subdivided as needed for the equllibration calculations. Some error 1s undoubtedly introduced by this procedure, but that error is difficult to quantify. The approximation is presumed to provide an effective compromise between the limitations of the unmodifled FRAS code and the complexity of a multivariate analysis, such as that of Esteves. 4

The modified code, called FRAS2, has been applied in several analyses to determine whether the results are consistent with experiments, and whether the detalled response of bubble distributions to thermal transients is as anticipated. Results of these analyses are presented in the next section. 


\section{RESULTS OF FRAS2 ANALYSES}

\section{A. Equilibration Effects in the H3 Transient}

A series of calculations has been carried out to 1llustrate the effects of equilibration for the TREAT $H 3$ translent, 6 which was analyzed in the original calibration of the FRAS code. 15 Figure 7 shows the results of four calculations

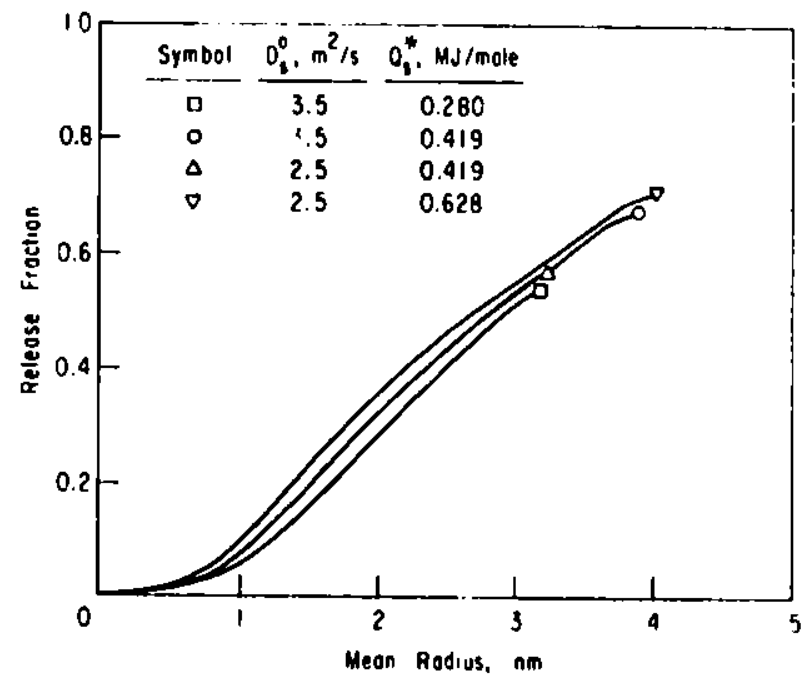

Fig. 7.

FRAS Results for the TREAT H3

Test, Assuming Different Surfacediffusion Coefficients and Heats of Transport.

with the FRAS code (assuming equilibrium bubbles) for two values of the surfacediffusion pre-exponent $D_{s}^{0}$ and three values of the heat of transport $Q_{8}^{*}$. The release fraction is plotted as a function of the mean bubble radius. A parametric analysis based on FRAS results has indicated a close relationship between the mean radius and the migration distance, wh1ch determines the release fraction. 16 This relationship varies slightly as the FRAS input parameters are varled, but only within a narrow range, as 1llustrated by Fig. 7. When $Q_{g}^{*}$ is Increased, the curve shifts slightly toward higher release fractions. When $D_{8}^{0}$ is Increased, however, the curve simply extends further along the same path.

Present information from the H3 posttest examination indicates a mean bubble radius of about $32.5 \mathrm{~nm}$ and a release fraction of about 0.8 for the radial node considered here. 6 This set of values corresponds to a point that would Ile above the curve in F18. 7. (The discrepancy from the original calibration of the BRAS code results from a more realistic value for grain size in the current version). Although these numbers are 11 mited in accuracy, 17 the discrepancy appears to be significant. 
Since a possible source of disagreement is the equilibration time, the FRAS2 code was used to calculate the gas release and mean radius as functions of time. The pre-exponent of the volume-diffusion coefficlent was varied by a factor of $10 \mathrm{higher}$ and lower than the nominal value [Eq. (13)] for these calculations. The results are shown in Fig. 8 .

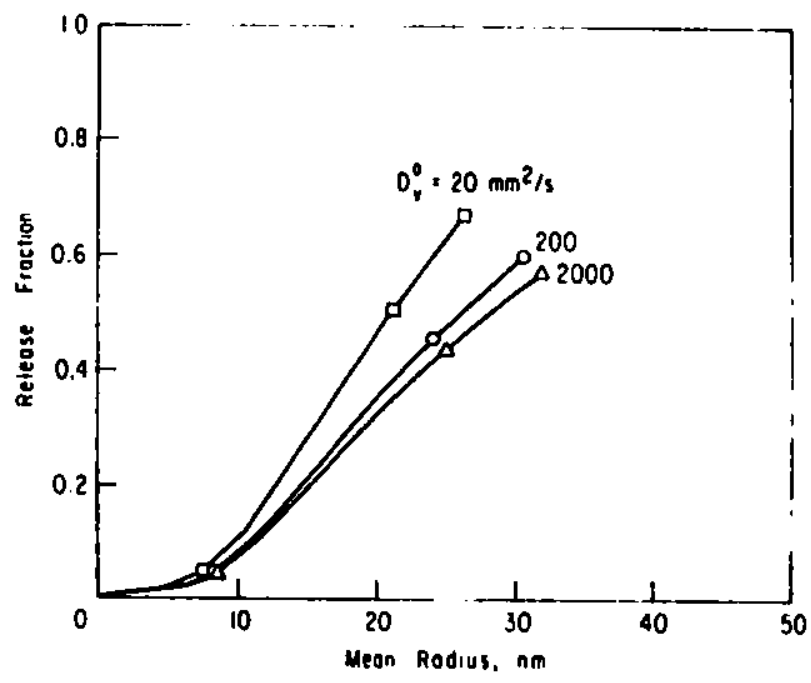

Fig. 8.

FRAS2 Results for the TREAT $H 3$ Test, Assuming Different Volumediffusion Coefficients.

The higher value gives complete equilibration throughout this transient, which involves fairly small bubbles. The results agree very closely with the corresponding curve in Fig. 7. The nominal and lower values result in nonequilibrium bubbles early in the transient. Release is enhanced for a given mean bubble size in these casis.

These results indicate that, for this particular set of conditions, the predicted equilibration behavior 18 in general agreement with the experimental results. Since the role of equilibration is relatively $8 \mathrm{ma} 1 \mathrm{l}$ here, because the mean Intragranular bubble size $18 \mathrm{small}$ and equilibration 18 rapld, the effects are not atrong enough to provide a quantitative benchmark test for the equilibration analysis of the FRAS2 code. However, the results of this comparison provide at least qualitative support for the need for, and the validity of, the analysis.

\section{B. Effects of Equilibration on Gas Behavior}

The basic difference between the results calculated with the FRAS2 code and the results from the equilibrlum analyels with the FRAS code can best be demonstrated by examining the differences in the predicted bubble-size distribut1ons. It is expected that: 
(1) the distribution will be narrower, since larger bubbles will not be able to equilibrate rapidly;

(2) there will be more release of gas to grain boundarles, since, in general, bubbles will be smaller, and hence more moblle;

(3) Intragranular swelling will be reduced, because of the finite rate of bubble growth; and

(4) there will be more, smaller intragranular bubbles, since coalescence probabilities will be reduced.

In each of these areas, the effect of equilibration must be determined quantitatively. A number of variables are 1mportant in quantifying these effects, including translent perlod, initial gas concentration, grain size, thermal gradient, and hydrostatic pressure. Only the first of these effects is consldered here.

A serfes of calculations was carrled out to illustrate the effect of the heating rate on the bubble-size distribution, on gas release to grain boundaries, and on intragranular swelling. Heating rates of 50,500 , and $5000 \mathrm{~K} / \mathrm{s}$ were considered, with other variables held constant. The values used were: grain size, $10 \mu \mathrm{m}$ (mean 11near intercept); Initial gas concentration, $1 \times 10^{17}$ atoms $/ \mathrm{mm}^{3}$; 1rittal temperature, $1500 \mathrm{~K}$; final temperature, $3040 \mathrm{~K}$; thermal gradient, $500 \mathrm{~K} / \mathrm{mm}$; and pressure, $0.25 \mathrm{MPa}$.

Figure 9 shows the end-of-transient cumulative bubble-size distributions for all six calculations. Logarithmic axes are used to cover the broad range

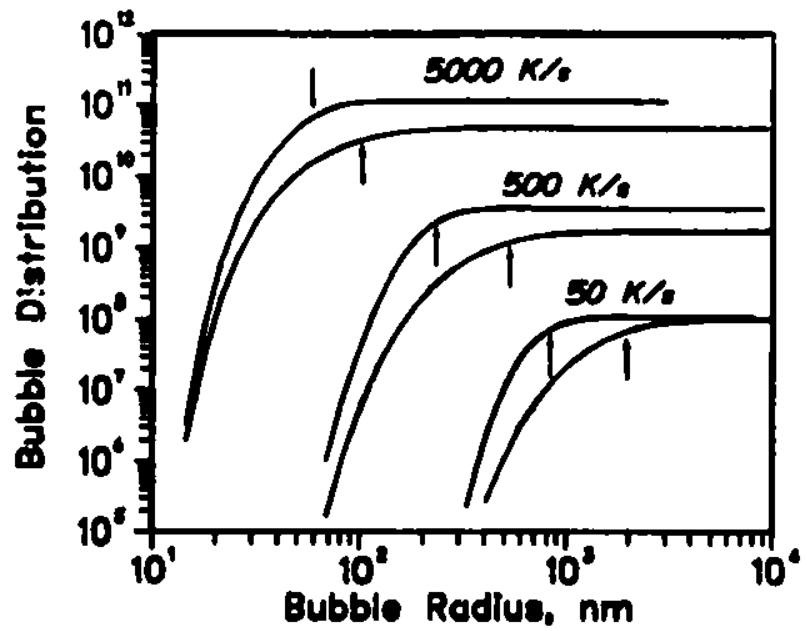

Fig. 9 . Comparison of Cumulative Bubblesise Distributions at the Solidus for Three Linear Thermal Ramps, Using FRAS and FRASZ.

of velues predicted. Bach distribution represents the number of bubbles per $\mathrm{mm}^{3}$ omaller than a particular radius. Several features sre apparent from 
this figure: (1) There is a difference in bubble density between FRAS and FRAS2 predictions that increases with the ramp rate; (2) the mean radius, denoted by sma11 vertical arrows in Fig. 9, is reduced by about a factor of 2 when equilibration is included; and (3) the FRAS distributions are broader than the FRAS2 distributions. Values of the bubble density and mean radius are tabulated in Table 1.

Table 1. Results of Heating-rate Comparisons

\begin{tabular}{|c|c|c|c|c|c|c|c|c|}
\hline \multirow{2}{*}{$\begin{array}{l}\text { Ramp } \\
\text { Rate, } \\
K / s\end{array}$} & \multicolumn{2}{|c|}{$\begin{array}{c}\text { Bubble Density, } \\
\text { Number } / \mathrm{mm}^{3}\end{array}$} & Mean & $\begin{array}{l}\text { Radius, } \\
\mathrm{nm}\end{array}$ & \multicolumn{2}{|c|}{$\begin{array}{c}\text { Release, } \\
\%\end{array}$} & \multirow{2}{*}{$\begin{array}{l}\text { Peak } \\
\overline{\text { FRAS }}\end{array}$} & \multirow{2}{*}{$\begin{array}{l}\begin{array}{l}\text { Swelling, } \\
\%\end{array} \\
\text { FRAS2 }\end{array}$} \\
\hline & FRAS & FRAS2 & $\overline{\text { FRAS }}$ & FRAS2 & $\overline{\text { FRAS }}$ & $\overline{\text { FRAS2 }}$ & & \\
\hline 50 & $1.0 \times 10^{8}$ & $1.1 \times 10^{8}$ & 1930 & 825 & 70.4 & 100.0 & 221 & 6.8 \\
\hline 500 & $1.7 \times 10^{9}$ & $3.5 \times 10^{9}$ & 524 & 234 & 32.6 & 62.9 & 198 & 10.6 \\
\hline 5000 & $4.6 \times 10^{10}$ & $1.1 \times 10^{11}$ & 106 & 61 & 15.0 & 21.7 & 66 & 10.9 \\
\hline
\end{tabular}

A IInear plot of the FRAS and FRAS2 results for the $500 \mathrm{~K} / \mathrm{s}$ thermal ramp is provided in Fig. 10. These distributions are very similar in shape, although

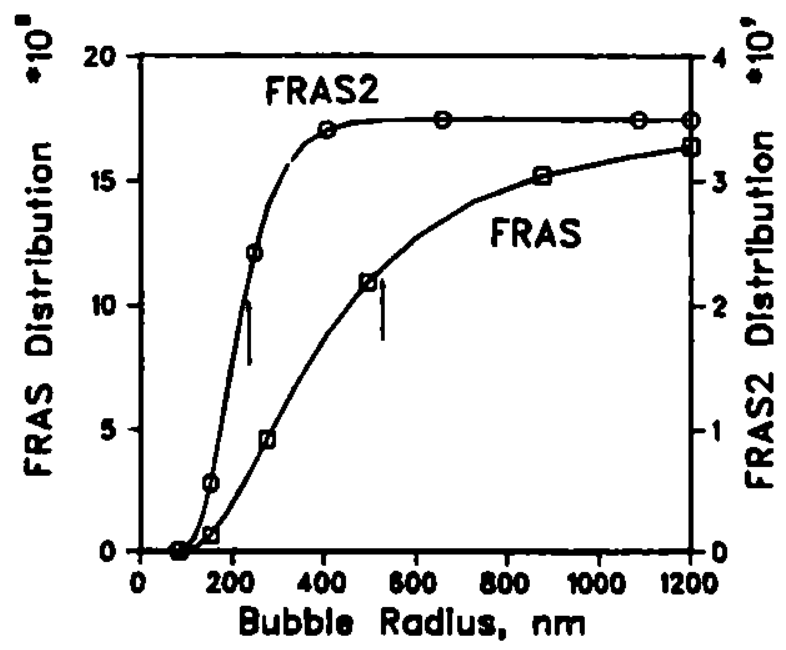

Fig. 10 .

Cumulative Bubble-size Distributions Calculated with the FRAS and FRAS2 Codes for a $500-K / 8$ Heating Rate.

not in magnitude, to those calculated for the other thermal ramps. The FRAS2 distribution is much narrower, with about the same minimum bubble size, but none of the larger bubbles predicted by the FRAS calculation.

The vertical arrows in F18. 10 Indicate the mean radil for the two calculations. The FRAS2 calculation predicts a mean radius less than half that predicted in the FRAS calculation. As a consequence, the gas release to grain boundaries is incressed by nearly a factor of two. Table 1 includes gasrelease results at transient termination for the three ramps. 
More detalled results of gas-release calculations are shown in Fig. 11 for FRAS calculations and in Fig. 12 for FRAS2 calculations. In both cases,
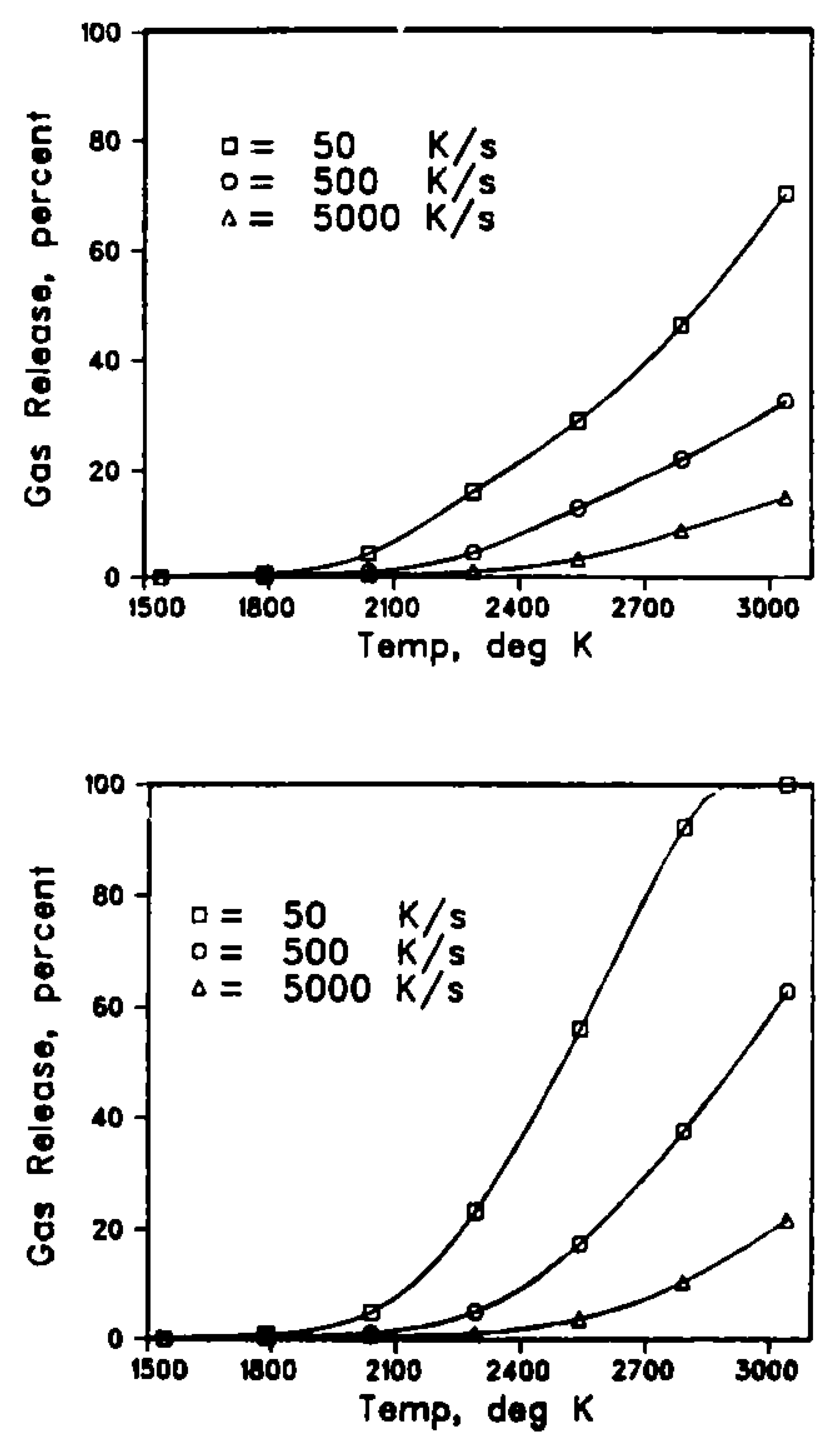

Fig. 11.

Gas Release to Grain Boundaries for Three Heating Rates, Calculated with the FRAS Code.
Fig. 12.

Gas Release to Grain Boundaries for Three Heating Rates, Calculated with the FRAS2 Code.

increasing the rate of temperature rise reduces the gas-release rate. The gas release calculated by the two codes is very similar up to about $5 \%$ release, which corresponds roughly to a mean bubble radius of $15 \mathrm{~nm}$. The curves subaequently diverge, with the least effect being seen for the fastest thermal ramp, corresponding to the smallest mean bubble sizes. Again, it is apparent that nonequilibrium effects are greater for larger bubbles.

Peak values of intragranular swelling are also 11sted in Table 1 . The major result is the large effect of absence of equilibrlum, which reduces the peak swelling by a factor of 30 in the slowest transient. More detalled resulte are shown in FIg. 13 for the FRAS calculations. Swelling decreases 


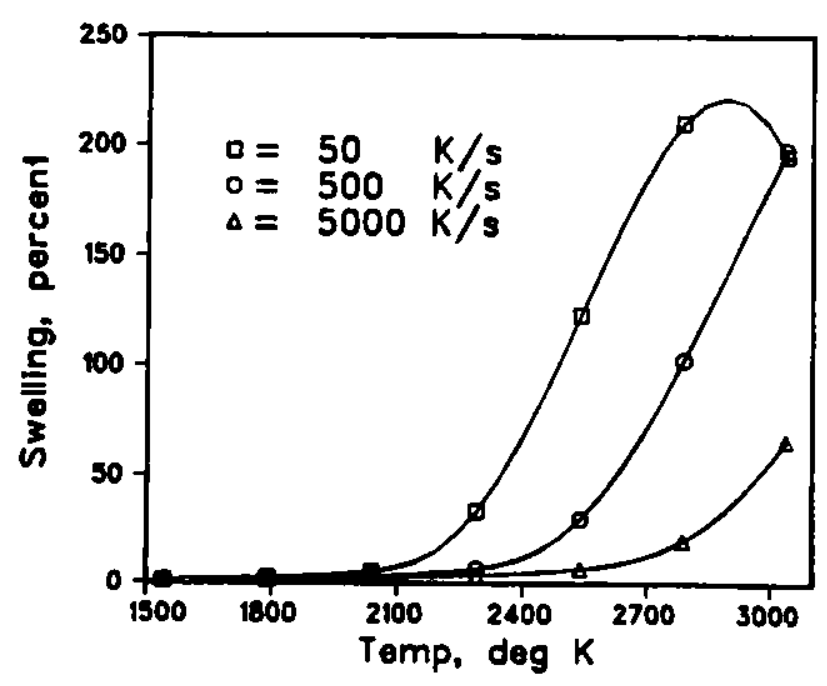

Fig. 13. FRAS-calculated Intragranular swelling for Three Heating Rates.

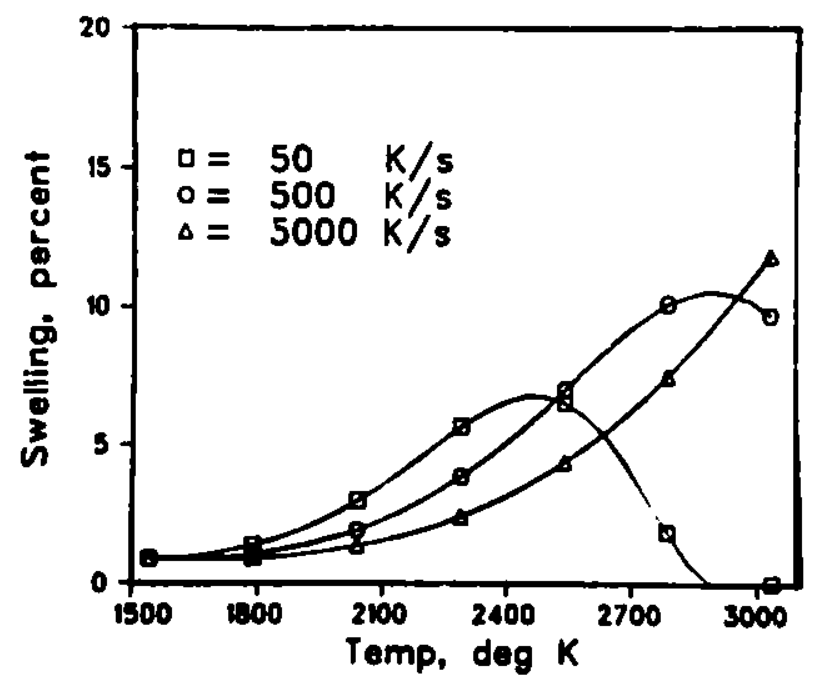

Fig. 14. FRAS2-caloulated Intragranular Swelling for Three Heating Rates. greatly as the ramp rate increases, but gross swelling is predicted even for the fastest ramp. Th1s swelling is beyond the level at which the analysis of swelling by collisional coalescence is considered valid. 3 The decrease in swelling at higher temperatures for the slowest ramp is caused by release of gas to the grain boundaries (compare F18. 11).

Figure 14 shows the intragranular swelling as modifled by nonequilibrium effects. The falloff in swelling occurs at lower temperatures than in the equilibrium calculations because release 18 more rapid, as shown In F1g. 12. The effect of thermal ramp rate 18 blightly less than in the FRAS calculation.

There is a fundamental difference in the owelling in the two calculations: In FRAS, swelling results directly and instantly from coalescence and temperature increases; In FRAS2, coalescence and changing temperature or pressure

only provide the nonequilibrium driving force, but swelling results from equilibration or diffusion of vacancies to bubbles.

\section{Dispersive Potent1al of Nonequilibrium Bubbles}

The preseure of fission gas in intragranular bubbles is generally higher than the equilibrium pressure, due to the finite time required for bubbles to expand to the equilibrium alze following coalescence or a temperature increase 
during a thermal transient. This overpressured gas constitutes a source of energy that can induce fuel disruption and dispersal in some situations. The potential work that the gas can perform on the surrounding fuel can be calculated In the FRAS2 code.

The potential work that can be performed by the gas as each bubble expands to its equilibrlum volume has two components: the work $W$ done against the radial stress in the fuel, and the work required to create additional bubble surface area. The dispersive potential $\mathrm{W}$ can therefore be expressed as the difference between the decrease in free energy of the gas and the Increase in surface energy. With the assumptions that the gas expansion is ideal, 1sothermal, and always at local equilibrium, the result for a given bubble 18

$$
W=m k T \ln \left(V_{f} / V_{1}\right)-\gamma\left(A_{f}-A_{1}\right)
$$

where $m$ is the number of $g a s$ atoms in a bubble, $V$ is the bubble volume, and $A$ 18 the surface area of the bubble. The subscripts 1 and $f$ refer to initial (nonequilibrium) and final (equilibrium) states, respectively. The dispersive potential is calculated in the FRAS2 code by suming the contributions from all bubbles in a unit initial volume.

It 18 also of Interest to character1ze the range of pressures to be expected and the potential volume increase. Clearly, if the overpressure in the bubbles 18 small or can be relieved by a small volume change, the dispersive potential will be small. Calculations were accordigly included in the FRAS2 code to determine the overpressure, weighted according to the number of gas atoms in each bubble-size class. The volume potential has also been calculated as the difference between actual, nonequilibrlum volume and the potential equilibrlum volume, essuming no additional coslescence.

Application of the FRAS2 code allowe a comparative evaluation of the potential for dispersive fuel motion from intragranular gas. For 1llustration, four casee have been considered (see Table 2): two calculated volding-driven transiente in the CRBR, corresponding to two different numerical representation of the reactor for computational purposes, and two thermal historles calculated for TREAT experiments. Although the overpreseures and potential volume increases are considerably different for the two CRBR transients, the dieperalve potential differe by only $10 x$. 
Table 2. Dispersive-potentlal Results from FRAS2 Calculations

\begin{tabular}{lccc}
\hline Case & $\begin{array}{c}\text { Overpressure, } \\
\mathrm{MPa}\end{array}$ & $\begin{array}{c}\text { Potentlal Volume } \\
\text { Increase, } \%\end{array}$ & $\begin{array}{c}\text { Work Potentlal, } \\
\mathrm{MJ} / \mathrm{m}^{3}\end{array}$ \\
\hline CR/N & 0.26 & 67 & 3.0 \\
CR/A & 0.15 & 104 & 2.7 \\
F1 & 0.03 & 35 & 0.5 \\
L5 & 0.17 & 27 & 1.2 \\
\hline
\end{tabular}

The dispersive potentials of the TREAT experiments indicate that the LS experiment Involved considerably greater potential for fuel dispersal than the F1 experiment. Experimental results are qualitatively consistent with this result. These results are ilmited to consideration of the effects of intragranular gas in promoting early fuel dispersal. It mist be recognized that grain-boundary separation, breakup into chunks, and even gross swelling are more 11kely to result directly from the effects of intergranular bubbles and pressurized porosity. However, the source of this gas 1s inevitably the Intragranular gas. 
IV. SUMTIARY AND CONCLUSIONS

An explicit, although simplified, treatment of the rate of bubble equilibration has been developed. Examples have been presented to indicate cond1tions under which nonequilibrium is important. The FRAS2 version of the FRAS code has been developed to include this treatment of bubble equilibration. Calculations have been carried out for a variety of cases to show the response of fisston gas to various transient conditions (e.g., various thermal ramp rates) when equilibration is considered. The general results of these calculations indicate a reduction in mean bubble size, an increase in intragranular bubble density, an increase in gas release to grain boundarles, and a major reduction in intragranular swelling, compared to the equilibrium results. These results are generally in agreement with expected behavior, based on experimental results, and indicate a significant improvement in modeling capab111ty.

The greatest model difference between the FRAS and FRAS2 codes is in the calculation of intragranular swelling. In the YRAS code, bubbles were assumed to equilibrate instantly in response to cosiescence or changes in temperature or pressure. Large, unrealistic changes in swellirg were of ten predicted. In the FRAS2 code, swelling becomes a rate effect. Coalescence or changes in temperature or pressure affect only the driving force for changes in bubble volumes. Actual changes occur by the diffusion of vacancles to or from the bubbles; this 18 the process that we have called equilibration, and it is the only means by which swelling 18 altered in the FRAS2 code.

The presence of nonequilibrium bubbles in the fuel comprises a source of energy that, under some conditions, may act to disperse the fuel. The dispersive potential of the flssion-gas bubbles has been quantified by considering the work that the expanding 8 as can perform against the stress in the material surrounding the bubble. Examples of this dispersive potential have been presented for several situations.

The work described in this report has been 1 imited to intragranular fission-gas behavior. The behavior of f18sion gas after it 18 relessed to grain boundaries 18 expected to play a wajor role in the response of Irradiated oxide fuel to a severe thermal transient. Work in progress will extend the present analysis to conetder the effecte of grain-boundary gas. 
Work in progress also includes efforts to determine a "best value" for the volume-diffusion coeffictent, by comparison of the experimental results with FRAS2 predictions. A major difficulty is that this coefficlent can vary by two orders of magnitude at $1773 \mathrm{~K}$ with a change in stolchiometry from 1.94 t.o 1.97 .18 There 18 some variation in stoichlometry with both burnup and pellet radius. 


\section{ACKNOWLEDGMENTS}

Helpful discussions with a number of colleagues are gratefully acknow1edged. These colleagues Include, but are not limited to, L. W. Deftrich, R. W. Ostensen, J. M. Kramer, R. J. DiMelf1, and G. Bandyopadhyay.

\section{REFERENCES}

1. F. A. Nichols, J. Nucl. Mat. 30, 143-165 (1969).

2. E. E. Gruber, J. Appl. Phys. 38, 243-250 (1967).

3. E. E. Gruber, Nuc1. Tech. 35(3), 617-634 (Oct 1977).

4. R. G. Esteves, Fission Gas Behavior During Fast Thermal Transients, $\mathrm{Ph} . \mathrm{D}$. Thes18, UCLA (1975).

5. J. M. Greisemeyer, W. G. Steele, D. Okrent, S. H. Chlen, and A. R. Wazzan, Trans. Am. Nuc1. Soc. 23, 174 (June 1976).

6. D. Stahl and T. H. Part1clan, ANL-8069 (Peb 1974).

7. J. Rest, ANI-76-121, 62 (Dec 1976).

8. C. Ronch1, "KInet1cs of F18sion Gas and Volatile FIssion-product Behavior under Transient Conditions in LWR Fuel," Appendix to ANL-75-72, LightWater-Reactor Safety Researoh Program: Quarterly Progrese Report, JulySeptember 1975.

9. W. L. Wang and R. N. Singh, ANL, private communication (1977).

10. B. W. Greenwood, A. J. B. Boreman, and D. E. Rimmer, J. Nucl. Mat. 4, 305-324 (1959).

11. B. E. Gruber, ANLRDP-52, 7.18 (Oct 1976).

12. G. Bandyopadhyay, ANI-KSD, private communication (1977).

13. C. A. BIneman and O. D. Slagle, HBDL, private combunications.

14. J. R. Katthew8, AERB-4-2643 (Sept 1974).

15. B. R. Gruber, ANL-8134 (Nov 1974).

16. R. W. Ostensen, ANL (presently Sand1a), private comminication (1976).

17. D. 8tahl, ANI-38D, private com unication (1976).

18. Af. Matske, GeK, Rarleruhe, private communication (1976). 